\title{
Translational Bioinformatics of HIV Peptide 2.0: From Dry To Wet Bench
}

\author{
Husna Nugrahapraja ${ }^{1,2,3^{*}}$, Alidza Fauzi $^{1}$, Alfonsus Adi Sadewa ${ }^{1}$, Azzania Fibriani ${ }^{1,3}$ Ernawati A. Giri-Rachman ${ }^{1,2,3}$ \\ ${ }^{1}$ School of Life Sciences and Technology \\ 2 Biosciences and Biotechnology Research Center \\ ${ }^{3}$ Research Center for Nanosciences and Nanotechnology \\ Institut Teknologi Bandung, West Java, Indonesia 40132
}

\begin{abstract}
Infectious diseases have become part of human civilization as a constant phenomenon throughout time. The Human Immunodeficiency Virus (HIV) is a virus that can cause immune system weakness by attacking cells that have CD4 receptors and CCR5 or CXCR4 chemokine coreceptors, namely helper T cells macrophages and dendritic cells. Therefore, people infected with this virus will be vulnerable to opportunistic infections and cancer. Here we proposed the translational bioinformatics approach from dry to wet bench. From proof of concept to the applications will be highlighted and discussed further in diagnostic of HIV and vaccine candidate development based on reverse vaccinology approach. We performed sequence data analysis to find the best epitope for HIV strains in Indonesia and compatible with the MHC class 1, the linear core with MHC class 2, and the B cell epitope. Epitope compatible with the two cells is expected to have high immunogenicity and potentially produce a response to good $\mathrm{T}$ memory. The epitope was then tested using an indirect ELISA against the serum of HIV positive patients. In this study, a negative control is used in blood serum from healthy individuals originating from Stored Biological Material and carried out with a commercial ELISA kit for the negative controls. The epitope in PBS buffer solution was coated to a microplate with an incubation time of 16 hours and various concentrations. As a result, the epitope from the concentration range of 1-2500 ng epitope/well can be recognized by HIV patient antibodies. In summary, the epitope from the in silico prediction had antigenicity against anti-HIV antibodies in sera samples and could also be a promising candidate for the vaccine. Keywords: B cell epitope, diagnostic, Reverse vaccinology, $T$ cell epitope, vaccine.
\end{abstract}

\footnotetext{
* Corresponding author: nugrahapraja@sith.itb.ac.id
} 